\title{
Clinical manifestations of diffuse large B-cell lymphoma in the skin and subcutaneous tissue a case series study
}

\author{
Katarzyna Dulik, Grażyna Kamińska-Winciorek, Ryszard Swoboda, Anna Kwiatkowska-Pamula, Sebastian Giebel
}

Department of Bone Marrow Transplantation and Oncohematology, Maria Skłodowska-Curie National Research Institute of Oncology (MSCNRIO), Gliwice Branch, Gliwice, Poland

Adv Dermatol Allergol 2020; XXXVII (5): 812-816

DOI: https://doi.org/10.5114/ada.2020.100494

Diffuse large B-cell lymphoma (DLBCL) is the most common subgroup of non-Hodgkin lymphoma, accounting for $30 \%$ to $40 \%$ of cases [1]. The most common symptom is lymphadenopathy (about 70\%) or involvement of other organs (30\%), including the gastrointestinal tract, skin and soft tissue, urogenital system, bones, respiratory system, thyroid, salivary gland, and breast. The presence of extranodal lesions is associated with a worse prognosis [2].

The paper presents 4 patients diagnosed with $\mathrm{DLBCL}$ with secondary involvement of the skin and subcutaneous tissue, treated at the Department of Bone Marrow Transplantation and Oncohematology, Maria Sklodowska-Curie National Research Institute of Oncology in 2017. In all cases the diagnosis was made on the basis of histopathologic examination with immunophenotyping of tumour sections from the internal organs, skin and subcutaneous tissue, and lymph nodes, affected by the disease process.

Case 1: A 68-year-old female patient with follicular lymphoma (FL) G2 diagnosed in April 2014 on the basis of histopathological examination of abdominal tumour located between the intestinal loops and the rectus abdominis muscle. Because of two disease relapses, the patient was treated with chemotherapy and radiotherapy, each time with a complete remission assessed by computed tomography. In March 2017, extensive oedema involving the skin and subcutaneous tissue appeared in the upper half of the right thigh, also affecting the right inguinal region. Despite the use of bendamustine monotherapy, there was rapid progression of isolated infiltrative lesions in the skin and subcutaneous tissue, initially located in the right thigh (Figure $1 \mathrm{~A}$ ), with subsequent hard infiltration on erythematous background, and numerous blisters with formation of ulcerations covered with bloody crusts (Figures 1 B, C), which was confirmed by computed tomography. The fifth line of treatment was administered according to the R-DHAP regimen (rituximab, cisplatin, cytarabine arabinoside, dexamethasone). Histopathological examination with immunophenotyping of cutaneous and subcutaneous tissue revealed transformation of FL to DLBCL, with BCL-2/c-Myc expression, corresponding to double-expressor lymphoma. The therapy used in this patient (four R-DHAP cycles) resulted in a significant reduction of tumour mass in the right thigh, with complete resolution of skin lesions (Figure $1 \mathrm{D}$ ). Unfortunately, during the preparation for bone marrow autotransplantation, there was a rapid recurrence in the form of massive infiltration in the right thigh and general symptoms of the disease. The patient died shortly afterwards.

Case 2: A 66-year-old female patient with oedema of the right lower limb that had increased for 1 year. Computed tomography examination of the abdomen and pelvis revealed extensive nodular infiltration in the lower abdomen and pelvis, soft tissue infiltration from the right groin to the right popliteal fossa along the adductor muscles of the medial part of the thigh. Histopathological examination with immunophenotyping of tumour in the right thigh confirmed the diagnosis of DLBCL (CD20+, BCL6-, BCL2+, MUM1+, Cyclin D1, CD3-, Ki67 about 90\%). Chemotherapy according to the R-CHOP regimen (rituximab, doxorubicin, vincristine, cyclophosphamide, prednisone) was administered. During this treatment, disease progression occurred with massive growth of a tumour within the subcutaneous tissue of the right thigh, up to $15 \times 10 \mathrm{~cm}$ in diameter, with increasing oedema of the right lower limb (Figure $1 \mathrm{E}$ ) and a tumour infiltrating the skin and subcutaneous tissue in the left shoulder joint, measuring $10 \times 10 \mathrm{~cm}$ in diameter (Figure $1 \mathrm{~F}$ ). Dermos-

Address for correspondence: Assoc. Prof. Grażyna Kamińska-Winciorek MD, PhD, Department of Bone Marrow Transplantation and Oncohematology, Maria Skłodowska-Curie National Research Institute of Oncology (MSCNRIO), Gliwice Branch, 15 Wybrzeże Armii Krajowej St, 44-101 Gliwice, Poland, phone: +48 32278 8523, fax: +48 32278 91 49, +48 604070208 , e-mail: grazyna.kaminskawinciorek@gmail.com Received: 20.03.2020, accepted: 6.04.2020. 

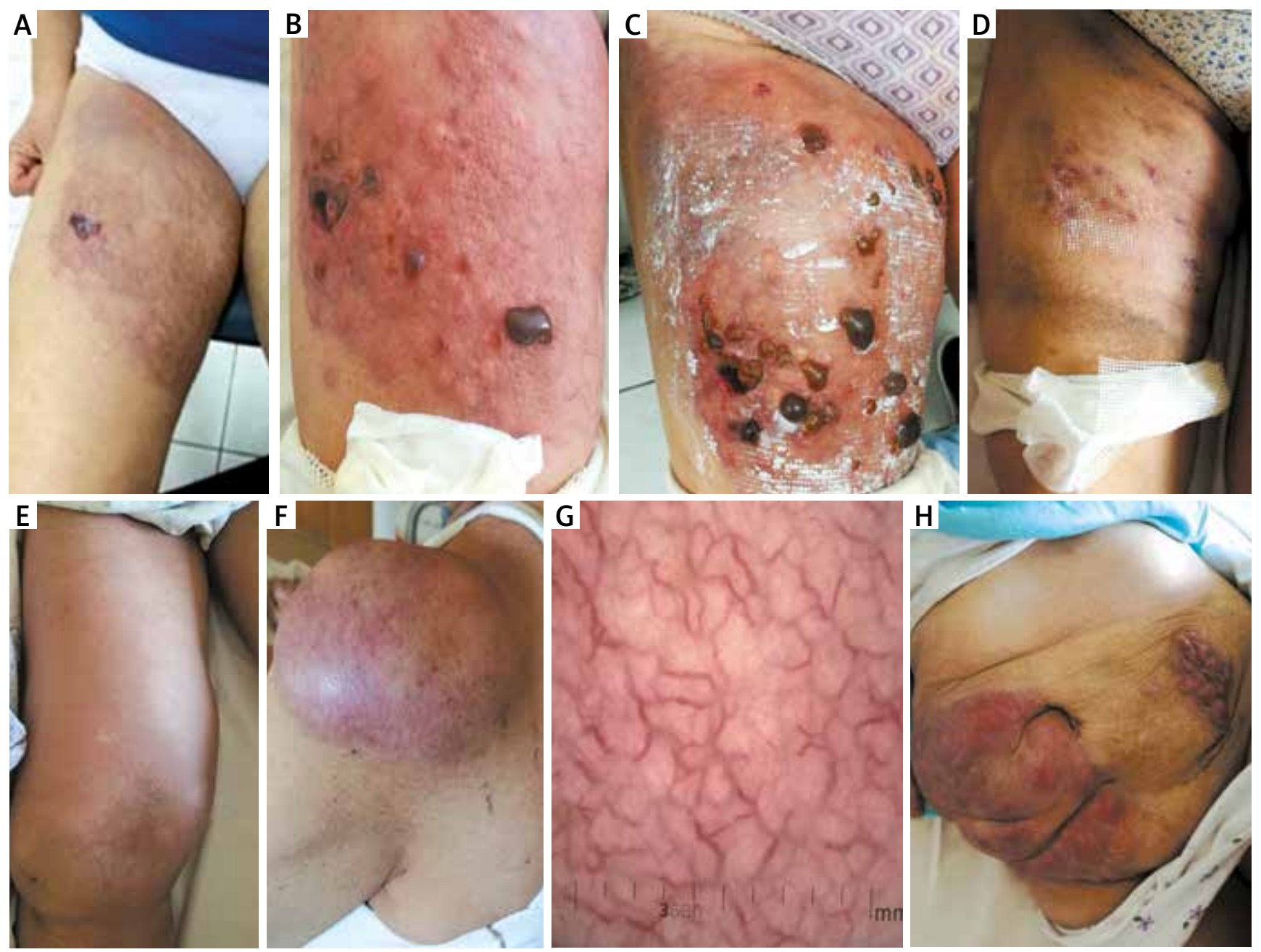

Figure $1 \mathrm{~A}$ - Purple red moderate infiltration on the upper anterior surface of the right thigh, quite well delimited, which led to the formation of blisters that quickly burst leaving an erosion, in the course of the DLBCL. B - Increasing oedema and coherent red infiltration in the entire thigh, with a tendency, only at this location, to form numerous large blisters up to $2 \mathrm{~cm}$ in diameter, filled with a bloody liquid. The presented clinical symptoms were the consequence of lymphoma (DLBCL) involving the subcutaneous tissue and the muscles of the right thigh. $\mathrm{C}$ - The local condition worsened with an increase in the number of blisters and a gradual increase in infiltration severity over the next 3 days in the course of the DLBCL. D - Clinical improvement with complete resolution of infiltration in the skin and subcutaneous tissue of the involved area confirmed by imaging examinations in computed tomography. The skin was soft, there were no infiltration, just discrete discolouration and numerous erosions in the epithelialisation phase were seen. $\mathrm{E}-\mathrm{A}$ large, coherent, non-painful tumour measuring $15 \times 10 \mathrm{~cm}$ in the right knee, with palpable infiltration along the adductor muscles of the medial part of the right thigh in the course of DLBCL. F - A large purple red tumour in the left shoulder area, with a network of numerous red telangiectasias visible through the skin. G - Dermoscopy revealed numerous serpentine linear telangiectasias of large cross-section and irregular distribution, located on a pinkish background. $\mathbf{H}-$ Progression of DLBCL lymphoma with involvement of the skin and subcutaneous tissue of the abdominal wall - purple, well-delimited, board-like infiltration is visible

copy showed the presence of numerous telangiectasias (Figure $1 \mathrm{G}$ ). The second line of treatment was followed by short-term CR of the new nodular lesions and partial remission of tumour in the right thigh. Despite 2 cycles of chemotherapy, tumour in the right thigh progressed with increasing swelling of the whole right lower limb. The third line of treatment was administered according to the $B G D$ regimen (bendamustine, gemcitabine, dexamethasone) with simultaneous irradiation of this lesion, without any tumour regression. In addition, new infiltrative lesions occurred in the skin and subcutaneous tissue of the abdomen (Figure $1 \mathrm{H}$ ).

Case 3: An 86-year-old female patient with DLBCL lymphoma (CD20+, CD3-, cyclin D1-, Ki67 about 50\%) in the left nasal cavity, diagnosed in 2012. She had been previously treated with 6 cycles of the R-COP regimen (rituximab, cyclophosphamide, vincristine, prednisone) with subsequent radical radiotherapy for residual lesions 

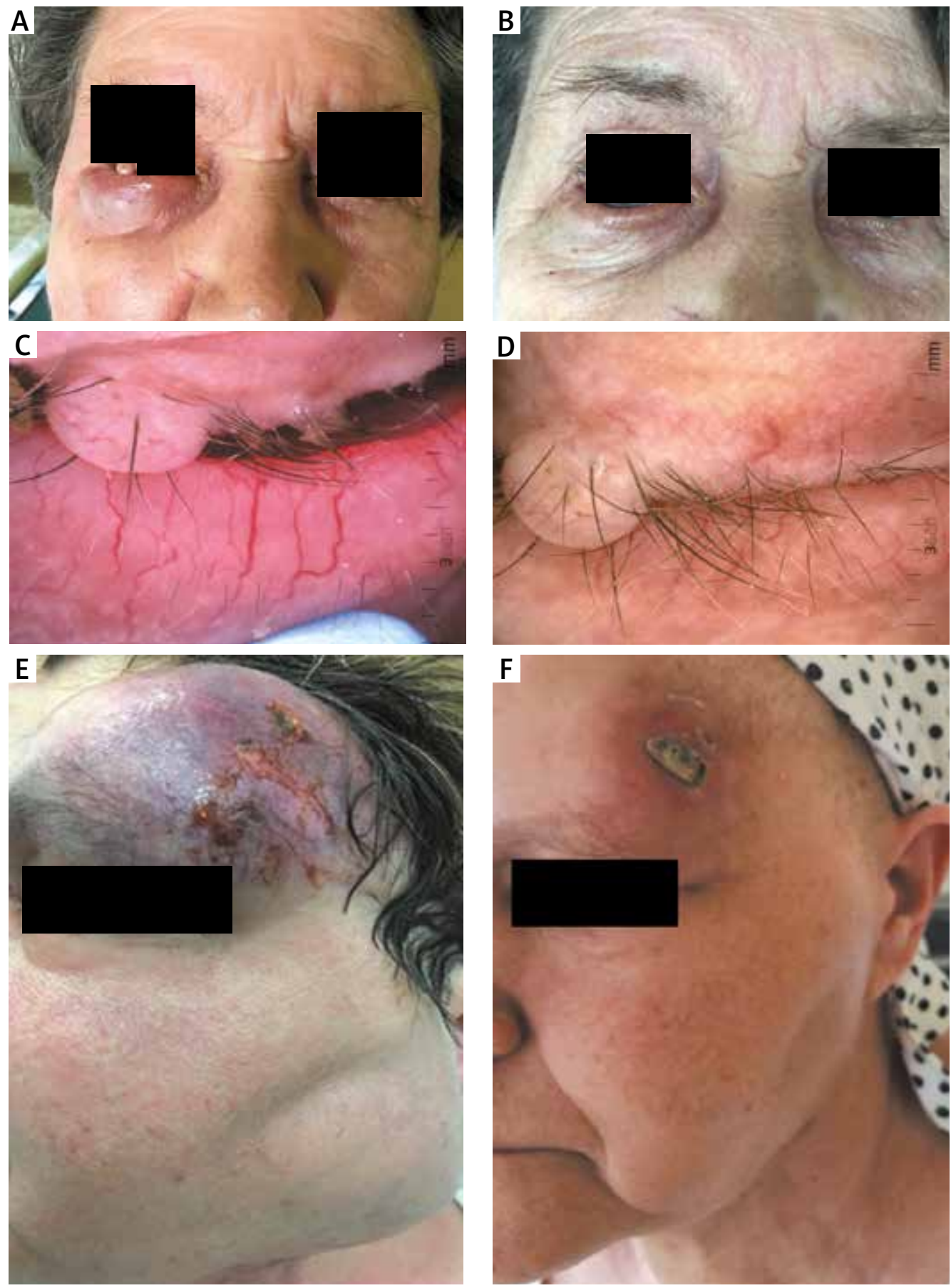

Figure 2. A - Clinical picture in the course of DLBCL with a hard infiltrative lesion and severe oedema of the right lower eyelid and narrowing of the palpebral fissure. B - DLBCL in the right orbit completely disappeared after first-line treatment with the R-COP regimen within 3 weeks. C - Dermoscopic image shows numerous telangiectasias (multiple, serpentine linear) in a parallel pattern, covering the entire dermoscopic field of view. $\mathbf{D}$ - Dermoscopic image of the lesion in the right lower eyelid after the treatment showed a significant reduction in the number and thickness of serpentine and arborising vessels in the examined area. $\mathrm{E}$ - Large nodular infiltration in the left orbit and the left frontal region - hard, cohesive, with a tendency to show complete lysis, and to form cyan-purple erosions. There is also a huge cervical lymph node cluster on the left side. F-DLBCL after 4 cycles of first-line treatment according to the R-CHOP regimen with partial remission of tumour in the left frontal area and lymph node remission 
(total dose 45 Gy), and the patient achieved the complete remission. In 2017, a rapidly growing hard infiltrative lesion of the right lower eyelid with accompanying oedema and infiltration in the suborbital region (Figure $2 \mathrm{~A}$ ) and in the right parotid salivary gland area were found. Due to the rapidly progressing disease, oral prednisone $1 \mathrm{mg} /$ kg was included, followed by R-COP chemotherapy with disease remission (Figure 2 B). The dermoscopic picture of the right lower eyelid lesion after the treatment showed a significant reduction in the number and thickness of serpentine-shaped and arborising blood vessels in the examined area (Figures 2 C, D).

Case 4: A 60-year-old female patient treated in April 2018 because of a rapidly growing tumour of the left frontal area and the left mandibular angle, without general symptoms (Figure $2 \mathrm{E}$ ), preceded by cervical lymphadenopathy persisting since August 2017. In February 2018, based on histopathological examination of a lymph node specimen from the left submandibular region, FL G3B (follicular pattern) was diagnosed with the following immunophenotype: CD20+, BCL2+, BCL6+, CD21+, CD30-/+, CD10-, MUM1-, CD3-, CD5-, CD23, MYC-, Cyclin D1-, CD138-, Ki67 proliferative activity up to $90-95 \%$, with suspected transformation to DLBCL. Positron emission tomography/ computed tomography scan showed active neoplastic lesions in the head and neck. The patient received $\mathrm{R}-\mathrm{CHOP}$ treatment with partial remission of tumour in the left supraorbital area and lymph node lesions (Figure $2 \mathrm{~F}$ ).

Non-Hodgkin lymphomas are neoplasms of the lymphatic system originating from the lymphoid cells of the $\mathrm{B}, \mathrm{T}$ and NK cell lines. The most common subtype is $\mathrm{DLBCL}$, which is often associated with extranodal lesions [3]. The most commonly affected organs include the gastrointestinal tract and the skin [4]. Secondary involvement of the skin occurs in approximately $25 \%$ of all skin lymphomas, of which $65 \%$ originate from B cells $(26 \%$ of these cases are DLBCL and $21 \%$ are $\mathrm{FL}$ ) [5]. Despite similar morphology, secondary lymphomas of the skin differ from primary lesions in terms of course, method of treatment, and prognosis [6]. Primary B-cell lymphomas of the skin have a milder course, while secondary lymphomas have a worse prognosis and are usually resistant to chemotherapy $[7,8]$. The primary DLBCL leg type represents approximately $5-10 \%$ of the B-cell cutaneous lymphomas. Skin lesions occurred as single, multiple, and even grouped [9]. Our own case series' study reveals that the clinical picture of presented B-cell lymphomas with secondary involvement of the skin is characterised by rapidly growing, non-pruritic nodular or extensive infiltrative lesions. It is therefore extremely important to know the clinical picture of lymphomas, and close monitoring and cooperation between an oncohaematologist, dermatologist and pathologist are necessary in order to implement the proper diagnostic and therapeutic management. Immunophenotyping has a diagnostic value in differentiating of the lymphoma subtypes [10], but does not always coincide with molecular studies (FISH). The most common cytogenetic changes in DLBCL concern BCL6 (30-40\%), BCL2 (15-30\%) and MYC (5-10\%). In a small percentage of cases these features may coexist, e.g. BCL2 and c-Myc ("double hit"), or additionally with BCL6 ("triple hit") [11]. These subtypes are commonly associated with extranodal lesions and resistance to treatment [12].

Patients with DLBCL lymphoma that is recurrent or resistant to immunochemotherapy have a poor prognosis [2].

Dermoscopic assessment may be helpful not only in the recognition of primary cutaneous B-cell lymphomas [13], but could be also implemented as a non-invasive diagnostic and monitoring tool for B-cell lymphomas with the secondary skin involvement [14].

Presented cases of non-Hodgkin lymphoma of the DLBCL type were characterized by their rapid progression and relapses, extensive secondary skin and subcutaneous tissue involvement, poor response to therapeutic regimens with the final bad prognosis. So far, only single cases of primary non-Hodgkin lymphoma with secondary involvement of the skin and soft tissues have been reported in the literature $[4,15]$. Treatment of this group of patients includes immunochemotherapy, new drugs, symptomatic treatment, and consolidation with allogeneic haematopoietic cell transplantation in selected cases.

Skin involvement in the course of lymphoma may be primary or secondary. It is worth noting that lymphomas with secondary involvement of the skin have a worse prognosis and are usually resistant to chemotherapy, which was unfortunately confirmed in the presented patients.

\section{Acknowledgments}

Katarzyna Dulik and Grażyna Kamińska-Winciorek participated equally in the development of this paper.

\section{Conflict of interest}

The authors declare no conflict of interest.

\section{References}

1. Raut LS, Chakrabarti PP. Management of relapsed-refractory diffuse large B cell lymphoma. South Asian I Cancer 2014; 3: 66-70.

2. Castillo JJ, Winer ES, Olszewski AJ. Sites of extranodal involvement are prognostic in patients with diffuse large B-cell lymphoma in the rituximab era: an analysis of the Surveillance, Epidemiology and End Results database. Am J Hematol 2014; 89: 310-4.

3. Møller MB, Pedersen NT, Christensen BE. Diffuse large B-cell lymphoma: clinical implications of extranodal versus nodal presentation - a population-based study of 1575 cases. Br J Haematol 2004; 124: 151-9.

4. Malkan UY, Gunes G, Yayar O, Demiroglu H. Diffuse large B cell lymphoma with extensive cutaneous relapse. Case Rep Med 2015; 2015: 137682 
5. Shamsudin N, Chang CC. Diffuse large B-cell lymphoma presenting with extensive cutaneous infiltration. Singapore Med I 2012; 53: e198-200.

6. Vargas TJ, Jorge SB, Gonzaga YB. CD30-positive cutaneous lymphoma: report of four cases with an emphasis on clinicopathological correlation. An Bras Dermatol 2017; 92: 86-91.

7. Kerl H, Fink-Puches R, Cerroni L. Diagnostic criteria of primary cutaneous B-cell lymphomas and pseudolymphomas. Keio J Med 2001; 50: 269-73.

8. Thomas V, Dobson R, Mennel R. Primary cutaneous large B-cell lymphoma, leg type. Proc (Bayl Univ Med Cent) 2011; 24: 350-3.

9. Gopal MM, Malik A. Primary cutaneous diffuse large b-cell lymphoma of the upper limb: a fascinating entity. Indian J Dermatol 2013; 58: 366-8.

10. Lenz G, Staudt LM. Aggressive lymphomas. N Engl J Med 2010; 362: 1417-29.

11. Gabeeva NG, Koroleva DA, Belyaeva AV, et al. Diffuse large B-cell lymphoma with concomitant C-MYC and BCL6 gene rearrangements with primary skin involvement: a case report and a review of literature. Ter Arkh 2017; 89: 85-92.

12. Sehn LH, Donaldson J, Chhanabhai M, et al. Introduction of combined CHOP plus rituximab therapy dramatically improved outcome of diffuse large B-cell lymphoma in British Columbia. J Clin Oncol 2005; 23: 5027-33.

13. Geller S, Marghoob AA, Scope A, et al. Dermoscopy and the diagnosis of primary cutaneous B-cell lymphoma. J Eur Acad Dermatol Venereol 2018; 32: 53-6.

14. Swoboda R, Kaminska-Winciorek G, Jaworska M, Giebel S. Dermoscopic follow-up of therapeutic response in mantle cell lymphoma with secondary involvement of the scalp. J Cosmet Dermatol 2018. doi: 10.1111/jocd.12826.

15. Lee WJ, Won $\mathrm{KH}$, Won CH, et al. Secondary cutaneous diffuse large B-cell lymphoma has a higher international prognostic index score and worse prognosis than diffuse large B-cel lymphoma, leg type. Acta Derm Venereol 2016; 96: 245-50. 